\title{
Reichweite und Nomenklatur der AAL-Technologien
}

\section{Pia Beyer-Wunsch}

Die Begrifflichkeiten Ambient Assisted Living (AAL) oder Active Assisted Living (AAL) sowie Smart Home finden sowohl in der Forschung und Wissenschaft als auch im allgemeinen Sprachgebrauch Verwendung. Es fällt dabei auf, dass mit wachsender Fülle an Einsatzfeldern und Technologien iterativ neue Begriffe zur Beschreibung der Systeme und Anwendungen generiert oder synonym verwendet werden. Beispielsweise werden „Smart Home“, „Smart Living“ oder „Connected Home“ synonym verwendet. Sie klingen ähnlich und weisen überlappende Wortfragmente auf, allerdings ist die Bestimmung des Einsatzes und damit der Anwendungsfelder unterschiedlich. Diese unscharfen, definitorischen Abgrenzungen verwischen eine exakte Zuordnung von Technologien und Anwendungsmöglichkeiten in ein oder mehrere Nutzungsfelder.

In diesem Beitrag wird ein möglichst umfassendes Bild der Nomenklatur aus dem Feld der assistierenden Technologien dargelegt, um dadurch die Vielzahl an Begriffen in ein Netzwerk zu übertragen sowie Gruppierungen an Begriffen zu erhalten, die in der Tat die gleiche Bedeutung haben, bzw. das inhaltlich Gleiche benennen.

\section{Assistierende Technologien}

Im technischen Kontext wird unter dem Begriff „Assistent“ eine Maschine, in Form von Hardware und/oder Software verstanden, die der Unterstützung von Personen in unterschiedlichen Lebenssituationen dient, welche sie nicht vollkommen autonom bewältigen können (Bendel, 2019, S. 14). Es sind helfende Geräte und digitale

\footnotetext{
P. Beyer-Wunsch (凶)

Institut für Datenbanken und Informationssysteme DBIS, Universität Ulm, Ulm, Deutschland

E-Mail: beyer@beyer-wunsch.de
} 
Technologien mit der Hauptaufgabe die Unabhängigkeit und auch die Funktionsfähigkeit von einzelnen Personen zu verbessern, oder auch den Erhalt zu sichern. Dabei soll insbesondere auch die soziale Teilhabe ermöglicht sowie das persönliche und individuelle Wohlbefinden gesteigert werden („Assistive devices and technologies“, who.int 2020). Durch diese Eigenschaften und Funktionen von technischen Assistenten ist es möglich, menschliche Beeinträchtigungen zu reduzieren, oder präventiv zu verhindern. Beispielhaft dafür stehen Telefonassistenten, Navigationsassistenten, Agenten in virtuellen Suchumgebungen mit intelligenter Hilfefunktion, Sprachassistenten oder virtuelle Assistenten wie Google Assistant, Siri, Alexa oder Cortana (Bendel, 2019, S. 14). Sie verfügen über Hilfefunktionen zum Beispiel für die Suche nach Informationen oder Personen, Hilfe zur Orientierung oder auch Hilfe zur Kontaktaufnahme von Personen.

In Abgrenzung zu den skizzierten digitalen, assistierenden Technologien stehen ferner eine große Anzahl an analogen, technischen Hilfsmitteln der Gesundheitsversorgung für Personen jeden Alters wie Anziehhilfen, Gehilfen, Badenwannenlifter, Rollatoren uvm. zur Verfügung.

Assistierende Technologien bieten eine große Varianz an Anwendungsmöglichkeiten. Diese umfassen ein Spektrum von hochspezialisierten Geräten für sehr spezifische Anwendungsbereiche bis hin zu Geräten und Software, die in einem sehr allgemeinen Kontext unspezifisch von einer großen Zielgruppe genutzt werden können. Unter diesen eher unspezifischen, alltäglichen Verbrauchertechnologien können unter anderem Computer, Telefone und Smartphones eingruppiert werden, welche als Assistenten für Menschen mit und ohne Behinderung oder Einschränkungen dienen können (Kylberg et al., 2015, S. 51-67).

Eine klare Definition von ,assistierenden Technologien“ "liegt bis heute nicht vor. Mitunter liegt die Ursache in der Polemik, inwiefern derartige assistierende Technologien auch dem „Human Enhancement“ zugeordnet werden können (Wolbring, 2011, S. 607-616). „Human Enhancement“ tangiert unter anderem die ethische Frage, inwiefern Technologien noch reine Assistenten sind, wenn durch sie die Möglichkeit besteht, menschliche Fähigkeiten über das natürliche menschliche Maß hinaus zu erweitern, und sie dann zu etwas zu befähigen, zu was sie von sich heraus nicht im Stande wären, so gesund sie auch sind (Ach \& Lüttenberg, 2013, S. 288 f.). Grundsätzlich ist im Kontext assistierender Technologien das Ziel von „Enhancement", die physischen und geistigen Eigenschaften eines Menschen zu verbessern, ganz unabhängig vom Zweck oder der Ursache des Einsatzes und von chirurgischen oder technologischen Anwendungsbereichen (Grundwald, 2008, S. 227 f.). Sollten Technologien dazu im Stande sein, die menschlichen Fähigkeiten auf ein übermenschliches $\mathrm{Ma}$ hinaus zu erweitern, muss geklärt werden, ob sie dennoch einen maßgeblichen, nachweisbaren medizinischen oder therapeutischen Zweck erfüllen, 
um dann möglicherweise über das Sozialsystem für Patienten finanziert werden zu können (Kylberg et al., 2015, S. 51-67).

\section{Smart Home}

Die Begrifflichkeit „Smart Home“, beinhaltet mit der Übersetzung und dem Sprachgebrauch in Deutschland eine Vielzahl an Bedeutungen. Dabei bedeutet der englische Begriff „,smart“ unter anderem „,clever“, ,klug“ oder auch ,,intelligent“. Im deutschen Sprachgebrauch wird der Begriff „,smart“ jedoch auch unter der Bedeutung wie „,geschickt“, ,pfiffig“ oder auch „,indig“" genutzt (OnlineWörterbuch Wortbedeutung.info, 2020). Synonyme für ,smart" sind unter anderem ,,aufgeweckt“, ,raffiniert“, ,geschickt“, ,,ausgefuchst“ oder auch ,,clever“(,,smart": duden.de 2020). Auffällig ist die Vielzahl an Bedeutungen und Synonymen für das Wort ,smart“ im deutschen Sprachgebrauch, wohingegen der Begriff ,,home“ deutlicher mit „zu Hause“, und weniger häufig mit „Heimat“ oder auch „Heim“ “im Sinne von „zu Hause“ abgrenzbar erscheint. Im Kontext von „Smart Home“ wird unter „,smart“ „,intelligent“, ,,vernetzt“, „,onlinefähig“ und „vernetzbar" verstanden. Auch hier liegt eine Vielzahl an Bedeutungen von ,smart" vor, die jedoch nur bedingt der ursprünglichen Bedeutung von ,smart“ entsprechen. Hingegen besteht bei „Home“ im Kontext von „Smart Home“ eine klare Bedeutung im Sinne von „zu Hause“, „Wohnung“, und „Haus“, was auch den Einsatzort von „Smart Home“ Technologien und Systemen angibt.

Der Begriff „Smart Home“ kann folgend mit ,intelligentes zu Hause“ übersetzt werden, bietet jedoch einen weiteren Raum für Synonyme. Darunter sind „Vernetztes Zuhause“, „Connected Home“ (Fokusgruppe Connected Home des Nationalen IT-Gipfels, 2014), und „Smart Living“, also ,intelligentes Leben“ zu nennen. Auch hier gibt der Begriff „Living“ den Einsatzort der Technologien an, so bezieht sich die Nutzung auf eine private Wohnumgebung.

Der Begriff „Smart“ steht für ein gewisses Maß an Intelligenz der Einzelgeräte oder auch des Systems, was möglichst auch intelligent in der Kommunikation mit dem Menschen agiert. Durch den Einsatz des Begriffs ,,intelligent“ und seinen Synonymen, wird der Wohnung, oder auch dem zu Hause die Eigenschaft der „Intelligenz“ zugeschrieben, was mit einem gewissen Maß an „Selbstständigem“ agieren oder reagieren im Sinne von Automatismus assoziiert (Wisser, 2018, S. 5) wird. Bekannt ist jedoch, dass die menschliche wie auch künstliche Intelligenz eine deutliche Spannweite an Fähigkeiten und Kenntnissen aufweist, was auch der Spannweite an tatsächlichen Fähigkeiten, betreffend der Automatismen von Geräten oder Systemen des „Smart Home“ entspricht.

Ähnliches gilt auch für ein weiteres Synonym von „Smart Home“, der „Gebäudeautomation“ (Wisser, 2018, S. 2). Die Begrifflichkeit „Automation“ stammt 
ursprünglich aus dem Griechischen und wird von dem Wort ,,automatos“ abgeleitet, was ,sich selbst bewegend“ bedeutet (Heidemann, 2013, S. 31). Auch wenn der Begriff der „Gebäudeautomation“ synonym zu „Smart Home“ genutzt wird, so liegt bei der „Gebäudeautomationen“ ein anderer Fokus der Funktionen und des Nutzens vor. Nach DIN EN ISO 16484-2 liegt dieser auf dem energieeffizienten und wirtschaftlichen Betrieb von Gebäuden, und der erhöhten Sicherheit (DIN - Deutsches Institut für Normung e. V., 2004).

Neben dieser Fokussierung soll die Automation auch den Komfort in der Wohnumgebung erhöhen, eine Alltagserleichterung bieten und auch Menschen mit Hilfebedarf unterstuitzen (Wisser, 2018, S. 5). Es ist zu sehen, dass gewisse Überschneidungen im Zweck und dem Ziel von „Smart Home“ und der „Gebäudeautomation" bestehen, diese jedoch nicht vollkommen deckungsgleich sind, was die synonyme Nutzung der Begriffe infrage stellt. Alle Einzelbegriffe im Themenfeld des „Smart Home“, wie „Connected“, „Smart“, „Home“ und „Living“ die in Kombination synonym genutzt werden, entsprechen damit dem Sinn und Zweck des Systems und der bereitgestellten assistierenden Leistungen. Im Kern sollen die Geräte des „Smart Home“ „aktiv“ untereinander in Kommunikation und Datenaustausch treten, um Arbeiten oder Umgebungsanalysen möglichst autonom und damit ohne weitere Anleitung oder Impuls eines menschlichen Nutzers bewältigen (Strese et al., 2010, S. 8-10; Frenken et al. 2013, S. 45-53). Mit diesen Eigenschaften werden die Geräte des „Smart Home“-Systems dem Anwendungsbereich des ,Internets der Dinge" und zugleich dem Themenbereich der Haus- und Geräteautomation zugeordnet.

\section{Nutzen und Ziele des Smart Home}

Wie bereits kurz erwähnt wird unter dem Begriff „Smart Home“ ein System für Zuhause verstanden, das aus mehreren homogenen oder heterogenen smarten Geräten besteht. Als grundsätzliche Zielsetzung von „Smart Home“ kann die Reduzierung des Energieverbrauchs von Geräten oder auch Hausanlagen, die Erhöhung der Sicherheit sowie die Steigerung des Wohnkomforts im häuslichen Umfeld bezeichnet werden (Wisser, 2018, S. 12). Auch soll „Smart Home“ dazu dienen, die Alltagsorganisation zu verbessern, und wenn möglich zu vereinfachen (VDE, 2010; Abicht et al., 2012, S. 25-102; Huning, 2000, S. 91-143), was zu einer erhöhten freien Zeit und dem Wohlbefinden beitragen kann. Weitere Ziele und Nutzen sind die Erhöhung der Betriebs- und Einbruchssicherheit sowie der Energieeffizient im Sinne von „Smart Grid“, mit beispielweise intelligenter Heizungsteuerung oder Klimaanlage in Kommunikation mit Markisen oder Jalousien für eine Best mögliche Ökobilanz (Bendel, 2019, S. 229). Möglich ist auch die intelligente und automatisierte Anpassung des Ressourcenverbrauchs von Strom oder Wasser mittels „Smart 
Metering" (Abicht et al., 2012, S. 25-102). Durch diese hohe Flexibilität an Systemen, Geräten und eingebundenen Technologien ist es grundsätzlich möglich, jedes Haus oder Wohnung mit „Smart Home“ Geräten auszustatten, welche zum Beispiel per WLAN oder Funk vernetzt sind und Sensoren Impulse für Aktionen geben. Die Grundlegende Steuerung und Einrichtung von gewünschten Parametern erfolgt über externe Steuerungsgeräte wie Smartphons oder Tablets.

Da die Kommunikationstechnologie in der Regel webbasiert ist, können die Daten mittels Webinterface bzw. APP mobil und auch von unterwegs abgerufen werden (Schelisch, 2015, S. 78 f.). Bei zahlreichen „Smart Home“ Systemen liegt keine Begrenzung der Aufgaben vor, da eine hohe Vernetzungsmöglichkeit unterschiedlicher Sensortypen, Funkschalter sowie Geräte unterschiedlicher Hersteller möglich ist (Wisser, 2018, S. 5). Wie hoch der Prozentsatz an smarten Geräten und Technologien sein muss, damit eine Wohnumgebung im Sinne des „Smart Home“ als vollständig vernetzt und oder automatisiert deklariert werden kann ist nicht klar benennbar, da hierfür keinerlei anerkannte Parameter vorliegen so Wisser (2018, S. 6). ,Smart Home“ bezieht sich dabei insbesondere auf intelligente Wohntechniken und Technologien mit Fokus auf Komfort und Sicherheit, allerdings wurden seit Beginn erster „Smart Home“ Geräte und Systeme auch zunehmend die Bereiche Kommunikation, Unterstützung sozialer Interaktion sowie Gesundheit bei der Entwicklung und Umsetzung von Assistenten betrachtet, da ein entsprechender Bedarf vorlag (Schelisch, 2015, S. 76-80).

\section{Ambient und Active Assisted Living}

Erst seit den 1990er Jahren und damit vergleichsweise spät werden Assistierende Technologien für die häusliche Umgebung im Sinne von Ambient Assisted Living in Deutschland näher betrachtet (Meyer et al., 1997, S. 93). Tiefere Konzepte zu Ambient Assisted Living wurden erst Anfang der 2000er Jahre entwickelt, die einen Übergang von „Smart Home“ zu „AAL“ darstellen. Das Konzept „Health Smart Homes (HSH)" verdeutlicht diese Entwicklung beispielhaft durch eine spezielle Anwendung für bequeme Häuslichkeit und Gesundheit, der Identifikation auffälliger Abweichungen von gewohnten Verhaltensweisen zum Schutz von Personen und der Früherkennung möglicher pathologischer Entwicklungen (Munstermann, 2015, S. 128).

In einer Bekanntmachung des BMBF 2008 zur Forschung von „Altersgerechten Assistenzsystemen für ein gesundes und unabhängiges Leben - AAL“ wurde erläutert, dass AAL mittels IT die Aufgabe und Funktion habe die Alltagsumgebung sowie die Pflege- und Gesundheitsversorgung im privaten Wohnumfeld zu verbessern (BMBF, 2008). Weitere Nutzen durch Informations- und Kommunikationstechnik sollen insbesondere im Alter dazu beitragen den Erhalt und auch Aufbau 
von sozialen Kontakten zu ermöglichen. Auch wird erläutert, dass die Zielgruppe u.a. aus alten, chronisch Kranken oder auch Risikopatienten besteht (BMBF, 2008).

Gersch und Liesenfeld (2012, S. 153) verstehen unter AAL technische und soziotechnische Konzepte, die einen Benefit durch technische Interaktion zwischen Umfeld und Individuum bietet, und die Lebensqualität erhöhen. Weiter führen sie aus, dass AAL sowohl aus altersneutraler wie auch alterspezifischer Perspektive betrachtet werden kann. Im Mittelpunkt von AAL steht auch für Georgieff (2008, S. 23) die Erhöhung der Lebensqualität mittels Informations- und Kommunikationstechnologien. Die Deutsche Gesellschaft für Biomedizinische Technik (DGBMT) und die Initiative Mikro-Medizin des Verbands der Elektrotechnik Elektronik Informationstechnik (VDE) legen den Fokus von AAL auf zu erhaltende Autonomie und Selbstständigkeit der Nutzer, welche ,sensibel und anpassungsfähig auf die Anwesenheit von Menschen und Objekten reagiert und dabei dem Menschen vielfältige Dienste bietet.“, und erweitert den Blick vom rein häuslichen Anwendungsbereich von AAL auch auf die Arbeit und die Freizeit (Becks et al., 2007, S. 3). Auch Becks et al., (2007, S. 3) erweitert den Anwendungsbereich von reiner Nutzung Zuhause, auf eine Nutzung in der Umgebung des Zuhauses, wie zum Beispiel im Garten oder anderen Außenbereiche nahe der Wohnung oder des Hauses. In einem weiteren Positionspapier geht der VDE auf das Entlastungspotential durch AAL für die „reife Gesellschaft“ (die Alten) ein, und erläutert, dass die Techniken des AAL eine Entlastung hinsichtlich ,Ermüdung, Überforderung und übergroßer Komplexität" bieten sollen, sowie die Technologien Einschränkungen im Alter ,weitgehend kompensieren“ (Ambient Assisted Living. Intelligente Assistenzsysteme im Dienst für eine reife Gesellschaft, o. J., S. 6).

Das Bundesministerium für Bildung und Forschung unterteilt AAL in vier Entwicklungsbereiche in Abhängigkeit des Nutzungskontextes. Überschneidungen in den Nutzungskontexten erschweren eine konkrete Trennung der Anwendungen, weswegen manche Anforderungen in mehr als einem Entwicklungsbereich aufgeführt werden. Diese Entwicklungsbereiche nach Lilgenau (2014, S. 67 f.) sind:

1. Gesundheit \& Homecare: Präventionstechniken und Dienstleistungen mit der Möglichkeit einer Fernüberwachung mittels netzbasierter Technologien, Funk oder WLAN. Zweck: Früherkennung eines potenziellen Verschlechterungszustandes; Sturzwarnsystem.

2. Sicherheit und Privatsphäre: Alarmfunktionen für Risikosituationen bei potenziell schädlichem Einfluss auf den Nutzer durch Feuer, Wasser und Gas. Automatisierte Notruffunktion, sowie Präsenz- oder auch Bewegungsmelder. 
3. Versorgung und Haushalt: Technologische Assistenten bei der Haushaltsorganisation und Haushaltsführung, sowie Ordnung und Reinigung. Intelligente Klimaanlage oder Haushaltsgeräte. Dieser Bereich weißt einige Eigenschaften des „Smart Home“ auf, da diese Anwendungsfelder auch altersunabhängig genutzt werden.

4. Soziales Umfeld: Kommunikationssysteme zur Kommunikation mit anderen Menschen mittels diverser Geräte sowie Geräte welche dem Erhalt der Mobilität älterer Menschen dienen. Im Mittelpunkt steht die Förderung von sozialen Kontakten und Beziehungen zur Prävention von verfrühter sozialer Isolation.

Auch Rocker und Ziefle (2012, S. 6-14, 42) gliedern die Anwendungsmöglichkeiten in Gruppen, wie dies das Bundesministerium für Bildung und Wissenschaft macht. Rocker und Ziefle Teilen in folgende Gruppen auf:

- General Support of Elderly (diverse Geräte zur Unterstützung im Alter ohne nähere Spezifikation),

- Medical Systems (Geräte und Systeme mit konkretem Gesundheits- oder Erkrankungsbezug),

- Intelligent Environments (intelligente Einrichtungsgegenstände wie Klimaanlagen oder Warnmelder) und

- Technical Infrastructures (Technische, vernetzte Systeme des Zuhauses).

Wie auch im Anwendungsbereich „Smart Home“, werden Geräte und Systeme des AAL für ähnliche Zwecke genutzt, jedoch liegt eine bedeutende Unterscheidung hinsichtlich der Zielgruppe und dem Anwendungsszenario vor. Im Bereich AAL dienen die Produkte wie Geräte oder Dienstleitungen, Konzepte, Technologien und Methoden der Alltagsassistenz von älteren Menschen, Menschen mit Behinderung oder auch pflege- und hilfsbedürftigen Menschen (Rosales, 2015). Im Zentrum von AAL stehen folglich explizit die besonderen Bedürfnisse dieser Zielgruppe, und AAL kann damit als besonderer Aspekt des „Smart Home“ angesehen und diesem untergeordnet werden.

\section{Nomenklatur Map}

Anhand der vorangegangenen Analysen von Begriffen aus den Themen- und Forschungsfeldern von „Smart Home“ und „AAL“, und der Betrachtung der Definitionen, lässt sich nun folgende Nomenklatur ableiten. Es besteht hierbei kein Anspruch auf Vollständigkeit. Die Nomenklatur ist in Form einer Map dargestellt (siehe Abb. 3.1), um Beziehungen der Themenfelder und der Begriffe, Hierarchien, Gemeinsamkeiten sowie Unterschiede optisch und strukturell darzustellen. Wie 


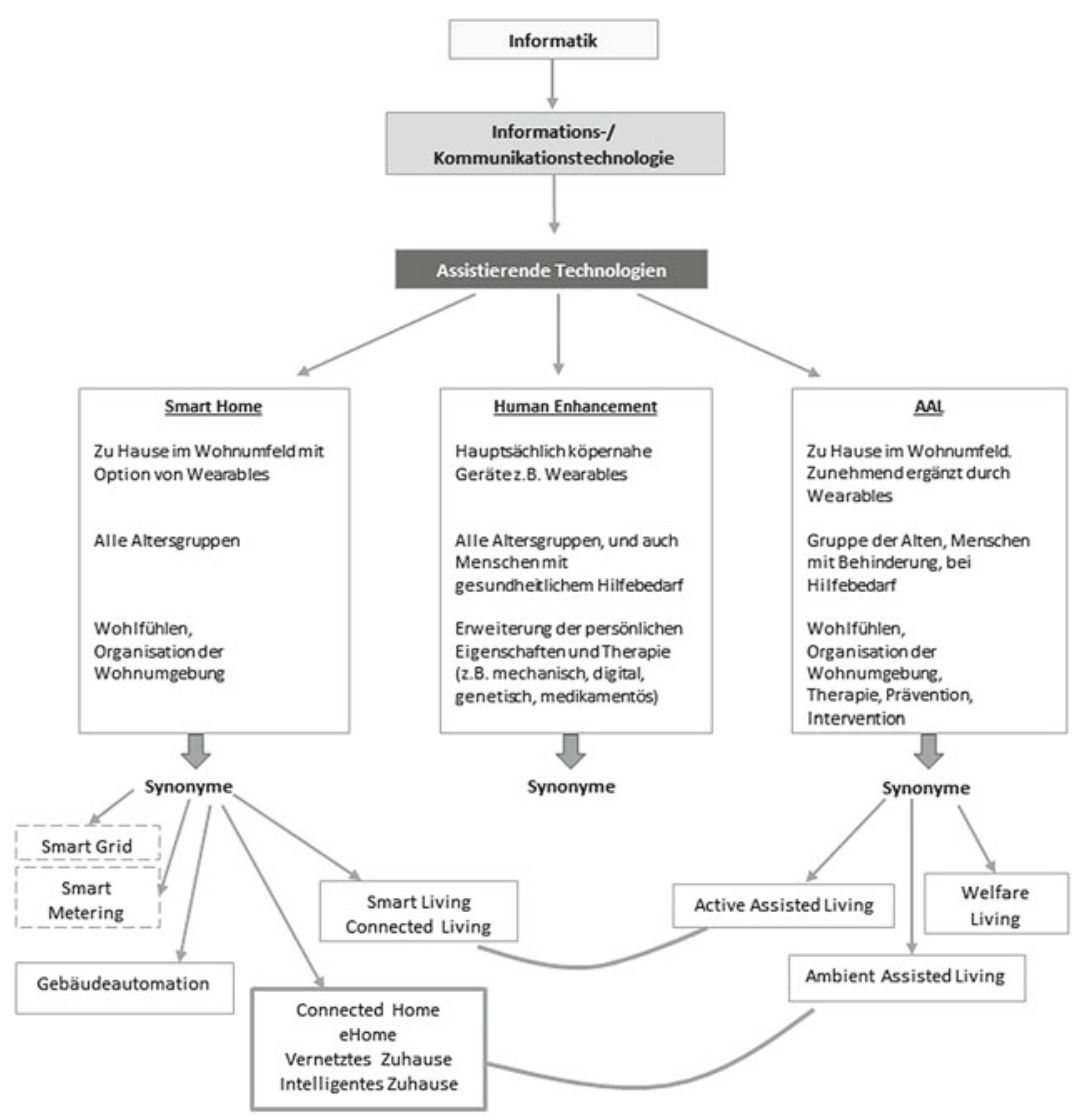

Spezifikations-Kontinuum:von Smart Home zu AAL

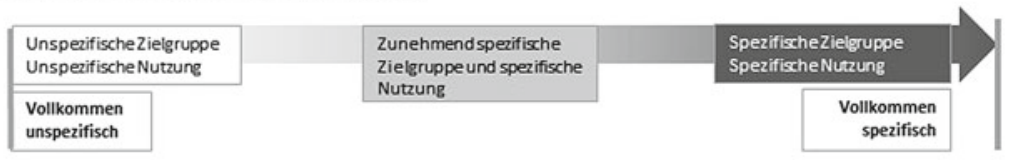

Abb. 3.1 Nomenklatur-Map. (Eigene Darstellung) 
bereits in den vorangegangenen Analysen zu den Begriffen, weisen die Felder hinsichtlich der Nutzungsumgebung, der Art der eingesetzten Geräte und Systeme wie auch der Zielgruppe Gemeinsamkeiten auf. Aus diesen Gründen werden in der Nomenklatur-Map beide Themenfelder aufgezeigt. Der Bereich des „Human Enhancement" stellt einen Übergang betreffend des Nutzenanlasses sowie des Bedarfes im Kontext Gesundheit und Therapie dar.

Die Nomenklatur ist hierarchisch aufgebaut, sodass die Themenfelder „Smart Home“, „Human Enhancement“ und „AAL“ eine klare Einordnung finden. Von oben nach unten wird die Zuordnung der betrachteten Themenfelder entsprechend konkretisiert.

Als ersten Oberbegriff wurde „Informatik“ gewählt, da die analysierten Themenfelder digitale und smarte Systeme sowie Geräte aufweisen. Diese Systeme und Geräte dienen der Kommunikation und Information des Menschen, beinhalten jedoch auch typische Teile der Informations- und Kommunikationstechnologien. Die Informations- und Kommunikationstechnologien (Abk. IKT) werden allgemein der Informatik zugeordnet bzw. untergeordnet. Zu den IKT gehören zum Beispiel Hardware und Software für Computer und Netzwerke, Radio, Fernsehen und auch Mobiltelefone.

Unter den IKT existieren wiederum diverse Geräte und Systeme welche als Assistenten für unterschiedliche Bedarfe genutzt werden können. Die Geräte können Hilfe und Unterstützung bei der Durchführung von Aufgaben und Herausforderungen bieten. Aufgrund dieser Eigenschaften ist es möglich die Devices als ,,assistierende Technologien" zu deklarieren.

Die Themenfelder „Smart Home“, „Human Enhancement“ und „AAL“ erfüllen den grundsätzlichen Zweck von assistierenden Technologien, nämlich Hilfe zu bieten und das Ziel Hilfe zu bekommen, weshalb sie in der Nomenklatur-Map den assistierenden Technologien zugeordnet sind.

Jedes Themenfeld hat jedoch einen unterschiedlichen, veränderten Fokus auf die Zielgruppe, den Nutzungskontext, und den Nutzungsanlass. In der vorangegangenen Analyse wurden insbesondere die Nomen und Adjektive der Synonyme der Begriffe „Smart Home“ und „AAL“ untersucht. Hier konnte festgestellt werden, dass für den Begriff „Smart Home“ unterschiedliche Begriffe als Synonym Verwendung finden. Auch wenn dies der Fall ist, so bestehen jedoch bei genauer Betrachtung dieser Unterschiede in der Auslegung der Bedeutung.

Dem Begriff ,Smart Home“ stehen am nächsten die Begriffe „Connected Home“, da „connect“ auch ein gewisses Maß an „smart“ erfordert. „Vernetztes Zuhause“ kann als direkte Übersetzung von „Connected Home“ angesehen, und „Intelligentes Zuhause“ als direkte Übersetzung von „Smart Home“. Der Begriff „eHome“, stehend für „,electronic home“ ist dem Begriff „Smart Home“ optisch eher fern, jedoch 
entsprechen die Eigenschaften von „eHome“ denen des „Smart Home“. Allen aufgeführten Begriffen gehört das Wort „Home“ an, welches den Einsatzort der Geräte und Systeme angibt.

Aufgrund der aufgeführten Gemeinsamkeiten, wurden diese Synonyme in der Map (siehe Abb. 3.1) zusammengefasst.

Zusammengefasst werden können auch die Synonyme „Connected Living“ und „Smart Living“, da sich diese im wörtlichen Sinn auf das „Leben“, oder eine Lebensweise mit assistierenden Technologien beziehen. Dies ist ein vollkommen anderer Blickwinkel des Einsatzes von assistierenden Technologien im Vergleich zu dem Synonymen, welche das Wort „Home“ beinhalten und sich auf den Einsatzort der Geräte und Systeme beziehen. Hiermit steht „Leben“, für etwas „Lebendiges“, „,bewegtes“" und „Home“ hingegen eher für „Beständigkeit“. Ein noch weiter von ,Smart Home“ entlehnter, synonymer Begriff, die „Gebäudeautomation“ weist durch das Wort „Gebäude“ auf den Einsatzort der Automation hin. ,Gebäude“ ist im Allgemeinen jedoch nicht uneingeschränkt mit Wohnumfeld, oder Zuhause gleichzusetzen, sondern bezeichnet etwas sehr viel unspezifischeres.

Der Begriff „Gebäude“ deutet auf etwas Funktionales, Objektives hin, auch wenn Gebäude durchaus Emotionen hervorrufen können. Zudem suggeriert das Wort „Gebäude" den Einsatz von assistierenden Geräten und Systemen eher in Bezug zu Haussteuerung, wie eingebauten Klimaanlagen oder fest integrierte Sicherheitssysteme. „Smart Home“ Geräte und Systeme werden dagegen eher in Wohnräumen als flexibel positionierbare Geräte angesehen, was im Gegensatz zur „Gebäudeautomation" steht. Aufgrund dieser Differenz wurde der Begriff separat unter den Synonymen aufgeführt.

Ähnlich ist dies auch bei dem Begriffen „Smart Grid“ (Intelligentes Stromnetz“ und „Smart Metering“ (intelligente Messung) welche beide dem Bereich des „Smart Home" zugeordnet werden, wie bereits erläutert. Jedoch sind diese Einsatzbereiche sehr speziell im Vergleich zu allgemeinen eher unspezifischen Einsatzbereichen der „Smart Home“ Geräte und Systeme, was einen deutlichen Unterschied zu dem Begriff „Smart Home“ darstellt. Daher stellt sich auch hier die Frage, ob die Begriffe „Smart Grid“ und „Smart Metering“ weiterhin als Synonyme für „Smart Home“ genutzt werden sollten?

Aufgrund der Unterschiede der im allgemeinen und fachsprachlichen Gebrauch genutzten Synonyme stellt sich nun die Frage, ob diese weiterhin als Synonyme angesehen werden können? Sowie auch unter welchen möglichen Bedingungen? Ebenfalls stellt sich hier die Frage, ob der Sinn, entsprechend der Eigenschaften der Geräte und Synonyme das vorherrschende Argument sind, die Begriffe weiterhin synonym verwenden zu können? Im Gegensatz zu dem Begriff "Smart Home“ liegen bei „AAL“ deutlich weniger Synonyme vor. Der Grund hierfür konnte jedoch nicht 
gefunden werden. Jedoch besteht die Möglichkeit, dass aufgrund der enger gefassten Zielgruppe und des Bedarfs sowie dem Einsatzweck weniger Möglichkeiten der Auslegung bzw. der Erweiterung bestehen.

Wie bereits im vorangegangenen Text und der Analyse der Synonyme erwähnt, wird die identische Abkürzung für die Begriffe „Ambient Assisted Living“ und ,Active Assisted Living“" genutzt. Diese Sachlage kann zu Verwirrungen darüber führen, ob unter den Abkürzungen das Gleiche zum Beispiel hinsichtlich der Zielgruppe, des Bedarfs der Geräte und Systeme verstanden werden kann. Diesbezüglich konnte festgestellt werden, dass dem so entspricht, und einzig unterschiedliche Begriffe genutzt werden. Wie bei „Smart Home“ bestehen auch im Bereich „AAL“ zwei Gegensätze in Bezug auf den Ausdruck von Aktivität und Einsatz der Geräte und Systeme in einer spezifischen Umgebung.

Dies drückt sich wie in der Analyse bereits ausgeführt durch die Worte ,ambient“ (Umgebung) und ,,active“ (aktiv, beweglich) in Kombination mit dem Begriff „Living“ aus. Der Begriff „Home“ findet bei keiner Wortkombination im Bereich AAL Anwendung, obwohl die Mehrheit der Geräte und Systeme zu Hause genutzt werden. Es scheint, als ob hier nicht der Fokus auf den Ort der Nutzung gelegt wurde, sondern der Fokus auf den allgemeineren Bereich, das Leben als solches liegt. Einzig durch das Wort ,ambient“ kann auf den Einsatz in einer (Wohn-)Umgebung hindeuten.

Der Begriff des „Welfare Living“ (Leben in Wohlbefinden) drück ähnlich wie der Begriff ,Active Assisted Living“ einen persönlichen Zustand aus, was dem Sinn der AAL Technologien entspricht. Diese sollen Hilfe bieten, unter anderem auch um Aktivität im Alter zu erhalten und ein Wohlgefühl zu erzeugen, in dem das Leben durch die Assistenz Erleichterung findet. Da damit auch ,Welfare Living“ dem Ziel und Zweck von „AAL“ entspricht kann es als Synonym in der AAL-Nomenklatur genutzt werden.

\section{Literatur}

Abicht, L., Brand, L., Freigang, S., Freikamp, H., \& Hoffknecht, A. (2012). Trends und Qualifikationsprofile durch das Internet der Dinge im Bereich ,Smart House“. In L. Abicht \& G. Spöttl (Hrsg.), Qualifikationsentwicklungen durch das Internet der Dinge. Trends in Logistik, Industrie und „Smart House“ (S. 25-102). Bertelsmann.

Ach, J. S., \& Lüttenberg, B. (2013). Human enhancement. In A. Grunwald \& M. SimonidisPuschmann (Hrsg.), Handbuch Technikethik (S. 288). Metzler. 
Becks, T., Dehm, J., \& Eberhardt, B. (2007). Ambient Assisted Living. Neue ,intelligente" Assistenzsysteme für Prävention, Homecare und Pflege. Frankfurt/Main: Deutsche Gesellschaft für Biomedizinische Technik (DGBMT) im VDE-Initiative Mikromedizin.

Bendel, O. (2019). 350 Keywords Digitalisierung (S. 264). Springer.

BMBF (2008). Bekanntmachung des Bundesministeriums für Bildung und Forschung von Richtlinien zur Förderung von Forschung und Entwicklung auf dem Gebiet „Altersgerechter Assistenzsysteme für ein gesundes und unabhängiges Leben - AAL“. https://www. bmbf.de/foerderungen/bekanntmachung-337.html. Zugegriffen: 18.07.2021

Der technisierte Lebensraum älterer Menschen Perspektiven zum technikgestützten Leben am Beispiel des Forschungsprojektes - „Ambient Assisted Shared Living for the Elderly“.

DIN - Deutsches Institut für Normung e. V. (2004). DIN EN ISO 16484-2:2004-10, Systeme der Gebäudeautomation (GA) - Teil 2: Hardware (ISO 164842:2004); Deutsche Fassung EN ISO 164842: 2004 35.240.99; 97.120 (2004-10) (35.240.99; 97.120) Berlin.

Fokusgruppe Connected Home des Nationalen IT-Gipfels. (2014). Vor dem Boom - Marktaussichten für Smart Home. (ANGA, BITKOM, GdW, ZVEH, ZVEI). https://www. bitkom.org/noindex/Publikationen/2014/Studien/Marktaussichten-fuer-Smart-Home/141 023-Marktaussichten-SmartHome.pdf. Zugegriffen: 15. Juli 2020.

Frenken, M., Hein, A., Herzog, O., Hoffmann, P., \& Müller, F. (2013). Länger selbstbestimmt Wohnen - Vernetzte Hausautomatisierung im Realeinsatz. In: VDE, AAL und Bundesministerium für Bildung und Forschung (BMBF) (Hrsg.), Lebensqualität im Wandel von Demografie und Technik. 6. Deutscher AAL-Kongress mit Ausstellung (S. 45-53). Tagungsbandbeiträge.

Georgieff, P. (2008). Ambient Assisted Living. Marktpotentiale IT-unterstützter Pflege für ein selbstbestimmtes Altern (S. 23). MFG Stiftung.

Gersch, M., \& Liesenfeld, J. (2012). AAL- und E-health-Geschäftsmodelle (S. 153-157). Gabler.

Grunwald, A. (2008). Auf dem Weg in eine nanotechnologische Zukunft. Philosophischethische Fragen (S. 227). Alber.

Heidemann, A. (2013). Nachhaltigkeit durch Gebäudeautomation. Auswirkungen von Gebäudeautomation auf die Nachhaltigkeit von Gebäuden im Lebenszyklus (S. 31). Stockach.

Huning, S., \& Wüstenrot Stiftung. (2000). Technik und Wohnen im Alter in internationaler Perspektive. In Wüstenrot-Stiftung (Hrsg.), Technik und Wohnen im Alter. Dokumentation eines internationalen Wettbewerbes der Wüstenrot-Stiftung (S. 91-143). Wüstenrot-Stiftung.

Kylberg, M., Löfqvist, C., Tomsone, S., Phillips, J., Liepina, Z., \& Iwarsson, S. (2015). A european perspective on the service delivery systems for assistive technology - Differences and similarities between Latvia and Sweden. Journal of Cross - Cultural Gerontology, 30(1), 51-67.

Lilgenau, A. (2014). Der technisierte Lebensraum älterer Menschen (Doctoral dissertation, Uni Wien), S. 67.

Meyer, S., Schulze, E., \& Müller, P. (1997). Das intelligente Haus, selbständige Lebensführung im Alter. Möglichkeiten und Grenzen vernetzter Technik im Haushalt alter Menschen: Bd. 30. Reihe Stiftung Der Private Haushalt (S. 93.). Campus.

Munstermann, M. (2015). Technisch unterstützte Pflege von morgen: Innovative Aktivitätserkennung und Verhaltensermittlung durch ambiente Sensorik (S. 33). Springer. 
Rocker, C. \& Ziefle, M. (2012). Current approaches to ambient assisted living. In INFORMATION TECHNOLOGY, L. N. (Hrsg.), Proceedings of the International Conference on Future Information Technology and Management Science \& Engineering (FITMSE'12) (Bd. 14, S. 6-14). IERI Press.

Rosales, B. (2015). Smart Home und Ambient Assisted Living (AAL). Forschung für ein sicheres und komfortables Wohnen und Leben. Begriffsbestimmung auf der Homepage des Forschungszentrums für Informatik (FZI) in Karlsruhe. http://www.fzi.de/forschung/for schungsfelder/detail/ffeld/smart-home-und-ambient-assistedliving-aal. Zugegriffen: 15. Juli 2020.

Schelisch, L. (2015). Technisch unterstütztes Wohnen im Stadtquartier: Potentiale, Akzeptanz und Nutzung eines Assistenzsystems für ältere Menschen (S. 76-78). Springer.

Strese, H., Seidel, U., Knape, T. \& Botthof, A. (2010). Smart home in Deutschland. http:// www.vdivdeit.de/publikationen/studien/smart-home-in-deutschland-untersuchung-imrahmen-derwissenschaftlichen-begleitung-zum-programm-next-generation-media-ngmdesbundesministeriums-fuer-wirtschaft-und-technologie. Zugegriffen: 28. Juli 2020.

Verband der Elektrotechnik Elektronik Informationstechnik (VDE). (o. J.). Ambient Assisted Living. Intelligente Assistenzsysteme im Dienst für eine reife Gesellschaft. Verband der Elektrotechnik Elektronik Informationstechnik.

VDE. (Hrsg.). (2010). VDE-Positionspapier. Intelligente Heimvernetzung: Komfort - Sicherheit - Energieeffizienz - Selbstbestimmung. Unter Mitarbeit von Thomas Becks, Birgid Eberhardt, Stefan Heusinger, Siegfried Pongratz und Johannes Stein. http://www.vde.com/de/Institut/Querschnittstechnologien/IntelligenteHeimverne tzung/Documents/Posipap-Heimvernetzung_Web\%5B1\%5D.pdf. Zugegriffen: 28. Juli 2020.

Wisser, K. (2018). Gebäudeautomation in Wohngebäuden (Smart Home): Eine Analyse der Akzeptanz (S. 2-5). Springer.

Wolbring, G. (2011). Hearing beyond the normal enabled by therapeutic devices: The role of the recipient and the hearing profession. Neuroethics, 6(3), 607-616.

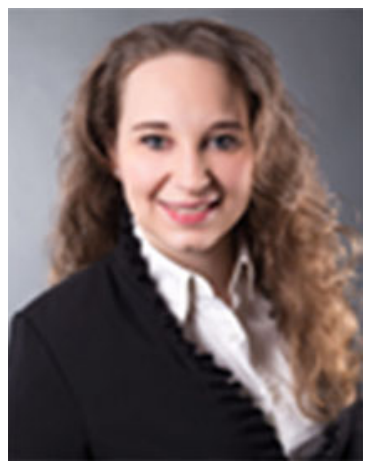

Pia Beyer-Wunsch Studierte Medien- und Kommunikationsmanagerin mit Abschluss Bachelor of Arts und Master of Arts. Bereits 2006 wendet sie sich den Fragen nach optimaler Voraussetzung für die Nutzung von Kommunikationstechnologien $\mathrm{zu}$ und führte entsprechende theoretische wie auch praktische Untersuchungen durch. Im Mittelpunkt stand stets die Nutzung durch Menschen mit besonderen Ansprüchen an die Technologie - Menschen nach Erkrankungen, in gesundheitlichen Akutsituationen, Kinder und Erwachsene mit Behinderung oder auch psychischer Erkrankung. Das Spannungsfeld zwischen Gesundheit, Medizin und Informatik ist ihr entsprechend bekannt und vertraut. Von 2018 bis 2020 war sie akademische Mitarbeiterin an der Dualen Hochschule Baden-Württemberg, am Standort Heidenheim im Studiengang Informatik. Seit 2020 ist sie Promovendin an der Universität Ulm (Fakultät 
Informatik, im Fachgebiet der medizinischen Informatik). Seit März 2020 ist sie Expertin und Mentorin des Solution Enabler Programms der Bundesregierung im Kampf gegen Covid-19. Sie ist Gründerin und Gründungsbegleiterin unterschiedlicher Start-Up's und seit 2012 Initiatorin sowie Gründerin der Initiative früh- und risikogeborene Kinder Kreis Heidenheim.

Open Access Dieses Kapitel wird unter der Creative Commons Namensnennung 4.0 International Lizenz (http://creativecommons.org/licenses/by/4.0/deed.de) veröffentlicht, welche die Nutzung, Vervielfältigung, Bearbeitung, Verbreitung und Wiedergabe in jeglichem Medium und Format erlaubt, sofern Sie den/die ursprünglichen Autor(en) und die Quelle ordnungsgemäß nennen, einen Link zur Creative Commons Lizenz beifügen und angeben, ob Änderungen vorgenommen wurden.

Die in diesem Kapitel enthaltenen Bilder und sonstiges Drittmaterial unterliegen ebenfalls der genannten Creative Commons Lizenz, sofern sich aus der Abbildungslegende nichts anderes ergibt. Sofern das betreffende Material nicht unter der genannten Creative Commons Lizenz steht und die betreffende Handlung nicht nach gesetzlichen Vorschriften erlaubt ist, ist für die oben aufgeführten Weiterverwendungen des Materials die Einwilligung des jeweiligen Rechteinhabers einzuholen.

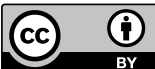

